\title{
Geologia
}

\section{Metacryphaeus rotundatus, um Novo Elemento da Fauna de Trilobites Calmoniidae (Phacopida), da Formação Ponta Grossa (Devoniano), Bacia do Paraná, Brasil}

\author{
Sabrina Pereira Soares' (spereirasoares@yahoo.com.br), \\ Marcello Guimarães Simões² (btsimoes@ibb.unesp.br), Juliana de Moraes Leme ${ }^{2}$ (leme@ibb.unesp.br) \\ 'Departamento de Educação - Faculdade de Ciências de Bauru - UNESP \\ Av. Engenheiro Luiz Edmundo Carrijo Coube 14-01, CEP 17033-360, Bauru, SP, BR \\ ${ }^{2}$ Departamento de Zoologia - Instituto de Biociências de Botucatu - UNESP, Botucatu, SP, BR
}

Recebido em 03 de outubro de 2007; aceito em 07 de novembro de 2007

Palavras-chave: Metacryphaeus rotundatus, Calmoniidae, Trilobita, sub-bacia Apucarana, Bacia do Paraná, Devoniano.

\section{RESUMO}

Trilobites Calmoniidae (Delo, 1935) constituem o grupo de Phacopida mais abundante e diversificado do registro fóssil da Formação Ponta Grossa, Devoniano, Sub-bacia Apucarana. Embora conhecidos desde o início do século passado, o estudo dos trilobites da Formação Ponta Grossa é ainda pouco abrangente, tanto do ponto de vista taxonômico, devido à concentração de estudos em certos táxons (calmoniídeos), como paleogeográfico e estratigráfico, em decorrência de concentração dos esforços de coleta nos afloramentos dos municípios de Ponta Grossa e Jaguariaíva. O exame de 398 espécimes, provenientes de rochas litoestratigraficamente correspondentes ao Membro Jaguariaíva, da Formação Ponta Grossa, especialmente da região de Tibagi - PR, revelou a presença de espécimes atribuíveis à espécie Metacryphaeus rotundatus (Kozlowski, 1923). Trata-se do primeiro registro desta espécie em rochas devonianas, do Brasil. Metacryphaeus rotundatus é uma espécie de ocorrência comum em rochas emsianas, da Formação Icla, da Bolívia. Embora no contexto do Domínio Malvinocáfrico, as afinidades paleobiogeográficas mais estreitas da fauna marinha sejam com as faunas das províncias Brasileira e Sul-africana, esse achado reforça outras evidências paleontológicas (e.g., conulários, trilobites homalonotídeos) indicativas da presença de elementos cosmopolitas, da Província Andina, nas assembléias fósseis do Devoniano da Bacia do Paraná. Tais formas viveram sob forte gradiente paleoclimático, desde clima temperado a temperado frio, até subpolar. Finalmente, no Devoniano paranaense, $M$. rotundatus ocupou, preferencialmente, fundos argilosos, ricos em matéria orgânica, depositados sob condições de águas plataformais, abaixo do nível de base de ondas de tempestades e indicativos de superfícies de inundação marinha.

Keywords: Metacryphaeus rotundatus, Calmoniidae, Trilobita, Apucarana sub-basin, Paraná basin, Devonian.

\section{ABSTRACT}

Calmoniids (Delo, 1935) are the most common and abundant group of trilobites of the fossil record of the Devonian Ponta Grossa Formation in the Apucarana sub-basin. Although known since the past century, the study of calmoniids has been taxonomically and stratigraphically biased. This is because some authors centered their studies on some particular genera from a few stratigraphic horizons within the basal portion of the Ponta Grossa Formation. The analyses of 398 specimens of calmoniid trilobites of the rocks of Jaguariaíva Member of the Ponta Grossa Formation, mainly from Tibagi county in the state of Paraná, Brazil, indicate the presence of specimens that are referable to Metacryphaeus rotundatus (Kozlowski, 1923). This is the first record of M. rotundatus in Brazilian Devonian rocks. Metacryphaeus rotundatus is a conspicuous species of the Emsian rocks of the Icla Formation, Bolivia. Although the affinities of the trilobite fauna of the Devonian Paraná Basin, in the context of the Malvinokaffric realm, are with the Brazilian and South Africa provinces, this finding is in accordance with new evidence (e.g., conulariids, homalonotids trilobites), indicating the presence of cosmopolitan species with Andean affinities. Metacryphaeus rotundatus lived in a broad paleoclimatic range, from a temperate, cold temperate to a subpolar climate. Finally, in the Devonian of the Paraná Basin, M. rotundatus lived and were preserved in muddy, organic rich bottoms, deposited in offshore waters, below the storm wave base, associated to marine flooding surfaces. 


\section{INTRODUÇÃO}

Calmoniídeos constituem o mais abundante grupo de trilobites do registro fóssil da Formação Ponta Grossa, Devoniano (Neolochkoviano-Emsiano, Dino, Oliveira, Antonioli, 1995; Grahn, Pereira, Bergamaschi, 2000; Gaugris e Grahn, 2006), sub-bacia Apucarana. Provavelmente devido à sua condição de preservação e abundância em determinados estratos, particularmente nas fácies pelíticas, geradas junto ou abaixo do nível de base de ondas de tempestades, estudos anteriores enfocaram, preferencialmente, às espécies desse grupo de Phacopida (Clarke, 1913; Popp, 1985; Carvalho e Edgecombe, 1991; Popp, Coimbra, Hauch, 1996; Carvalho, Edgecombe, Lieberman, 1997). Embora esses estudos tenham sido iniciados em 1913, com a clássica monografia de J. M. Clarke ele é ainda tendencioso, em parte, porque a informação disponível está maiormente restrita a algumas ocorrências “clássicas” de rochas devonianas, dos municípios de Jaguariaíva e Ponta Grossa, PR (Clarke, 1913; Popp, 1985; Popp, Coimbra, Hauch, 1996). Em menor grau, têm sido examinadas as ocorrências da região de Tibagi - PR, cuja duplicação e retificação do traçado de algumas rodovias têm levado a novas descobertas paleontológicas (Bosetti, 2004).

No presente estudo, são analisadas as novas ocorrências de calmoniídeos encontradas em folhelhos e siltitos da porção basal da Formação Ponta Grossa, aflorantes na estrada de rodagem PR-340, Tibagi e na estrada que parte de Tibagi em direção NW, rumo a Telêmaco Borba (Bosetti, 2004; Ghilardi, 2004), complementada pelo re-exame de extensa coleção de trilobites provenientes dos clássicos afloramentos da Formação Ponta Grossa da região de Jaguariaíva, PR (Petri, 1948; Lange e Petri, 1967; Petri, 2006). Durante o exame dos trilobites dessas localidades foi verificada a ocorrência de uma espécie do gênero Metacryphaeus Reed (1907), ainda não referida à fauna de calmoniídeos do Brasil. Em conjunto com as outras espécies de trilobites já descritos para a Formação Ponta Grossa, essa nova ocorrência tem importância paleobiogeográfica e geocronológica. Dessa forma, o presente estudo tem por objetivo:

1. a descrição dessa nova ocorrência de espécie do gênero Metacryphaeus Reed (1907), no Devoniano paranaense;

2. o entendimento de suas implicações paleobiogeográficas.

\section{MATERIAL E MÉTODOS}

\section{Material examinado, procedência e posição estratigráfica}

A Formação Ponta Grossa é caracterizada por sucessão monótona de argilitos intensamente bioturbados, folhelhos, siltitos e lentes de arenitos, registrando depósi- tos marinhos de plataforma interna e externa. Para o topo da formação, predominam os folhelhos negros, anóxicos, transgressivos, sendo escassa a ocorrência de macroinvetebrados marinhos. Os espécimes estudados, num total de 398 exemplares, são provenientes de rochas correspondentes, litoestratigraficamente, ao Membro Jaguariaíva (Petri, 1948; Lange e Petri, 1967; Petri, 2006), aflorantes nos municípios de Tibagi e Jaguariaíva, no Estado do Paraná (Figura 1).

Em Tibagi, as coletas foram conduzidas ao longo da seção aflorante na PR-340, que liga os municípios de Castro e Tibagi, bem como na estrada que parte de Tibagi, em direção NW, rumo a Telêmaco Borba (Figura 2A) (Bergamaschi, 1999; Bosetti, 2004; Ghilardi, 2004). O afloramento da PR-340 está situado no km 60, referente ao intervalo basal da Formação Ponta Grossa e é constituído por arenito fino, siltitos cinza-médios micáceos, fossilíferos, representando depósitos litorâneos de foreshore e shoreface (Bergamaschi e Pereira, 2001). Já o afloramento da estrada Tibagi-Telêmaco Borba está situado nas proximidades (cerca de $3 \mathrm{~km}$ ) da cidade de Tibagi. A espessura total da seção é de aproximadamente $240 \mathrm{~m}$, estendendo-se por aproximadamente 15 km (Bergamaschi, 1999). Porém, para esse estudo as coletas restringiram-se ao intervalo até $70 \mathrm{~m}$ da base da seção, referente aos estratos equivalentes ao Membro Jaguariaíva, da Formação Ponta Grossa (vide Bergamaschi, 1999). Nessa seção, os litotipos são representados por folhelho ou siltito cinzamédio a escuro, laminado a bioturbado, que grada para siltito, com interlaminações delgadas de arenito muito fino. No topo, observa-se o aparecimento de estratos formados por arenitos muito finos e finos, com estratificação cruzada hummocky (Bergamaschi e Pereira, 2001) (Figura 2A). Os trilobites calmoniídeos foram encontrados, respectivamente, a 8 e 48 m da base da Formação Ponta Grossa, em argilitos a siltitos, por vezes maciços, que registram eventos de inundação marinha (Bergamaschi e Pereira, 2001; Ghilardi, 2004).

Em Jaguariaíva, os exemplares de calmoniídeos foram coletados nos intervalos estratigráficos a 17, 30, 33, 38, 44 e $48 \mathrm{~m}$ da base da Formação Ponta Grossa, respectivamente, nos km 2,2 e 6,0 do corte da estrada de ferro JaguariaívaArapoti (Figura 2B). Porém, exemplares de Metacryphaeus rotundatus foram encontrados apenas a $48 \mathrm{~m}$ da base da Formação Ponta Grossa. Nesses intervalos estratigráficos predominam os horizontes representados por depósitos de sufocamento ou tempestitos distais (Simões et al., 2000a, 2000b; Rodrigues, Leme, Simões, 2003), associados a eventos de inundações marinhas (Bergamaschi e Pereira, 2001), que são progressivamente sucedidos por folhelhos cinza escuros e siltitos com delgadas intercalações de arenitos, intensamente bioturbados (Bergamaschi e Pereira, 2001). 


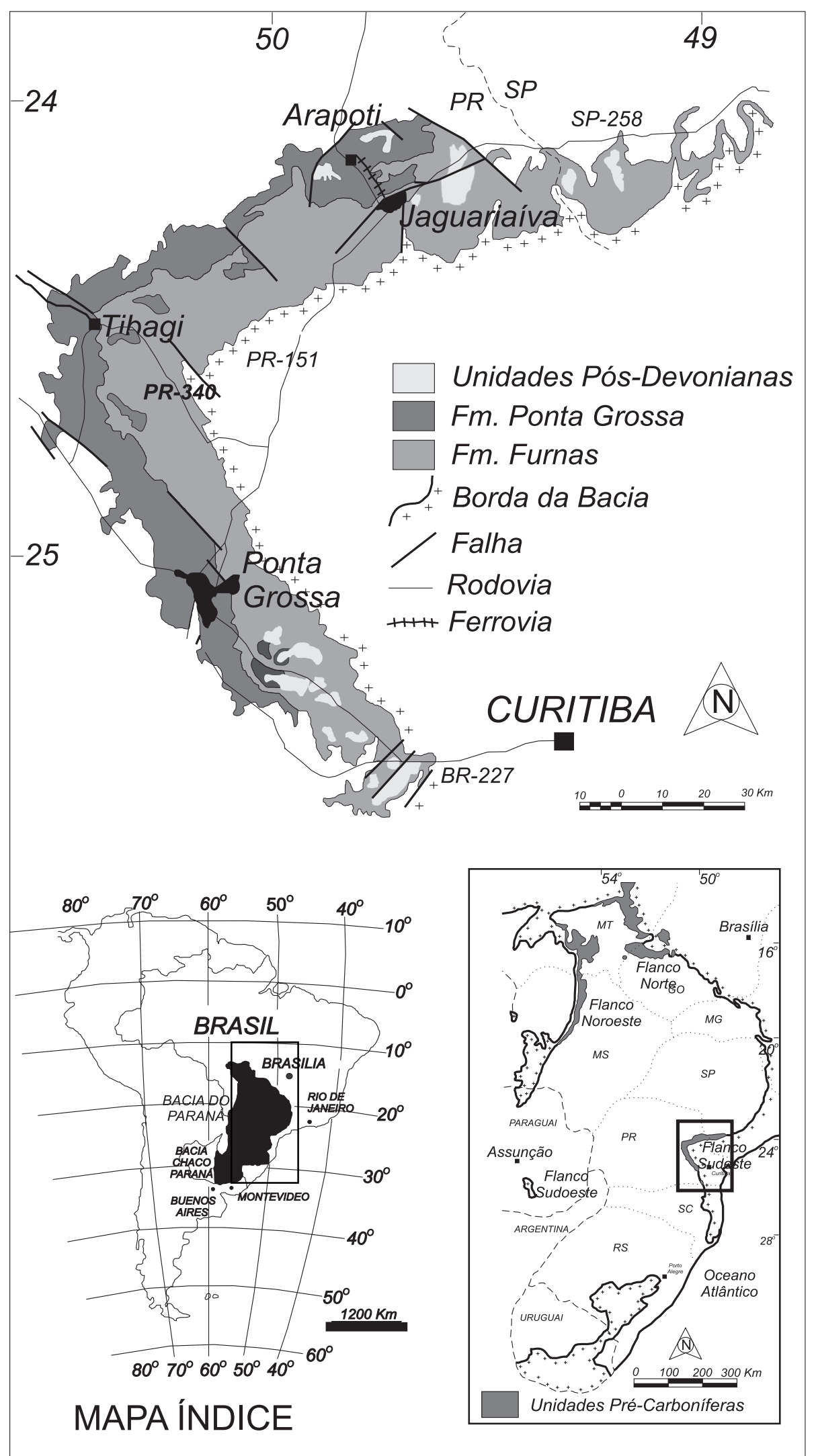

Figura 1. Mapa de localização dos afloramentos fossilíferos estudados, na Formação Ponta Grossa, mostrando a faixa de afloramentos, da borda leste da Bacia do Paraná. 
A

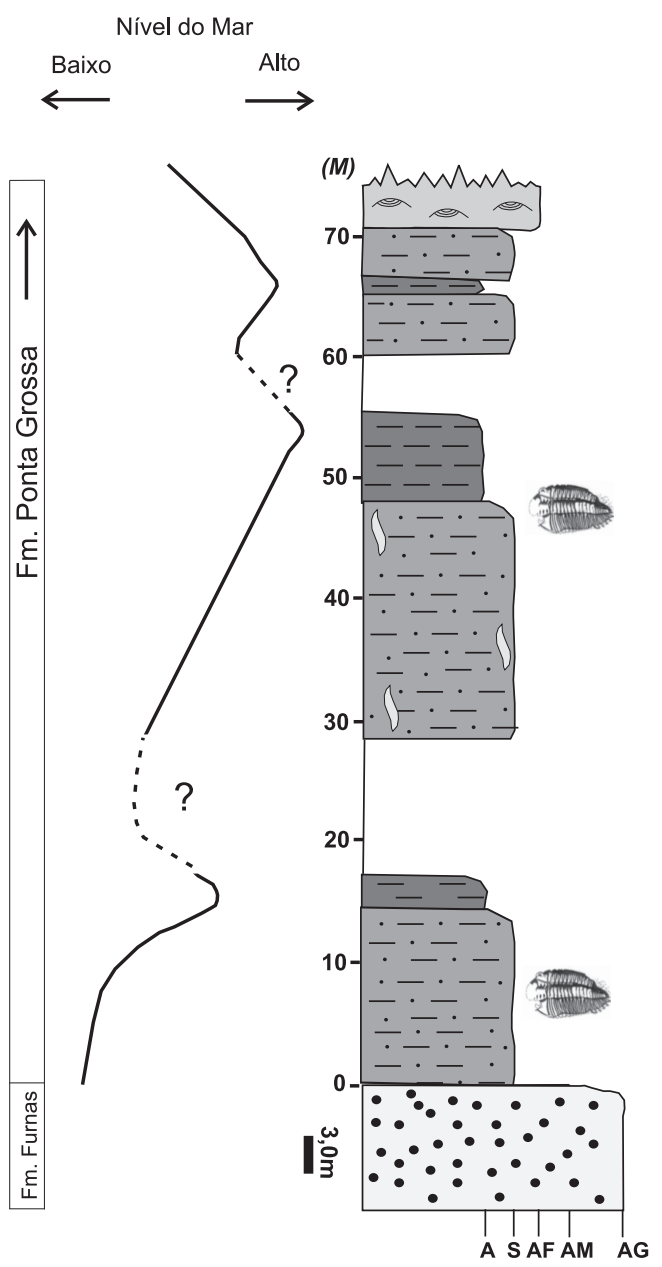

B Nivel do Mar

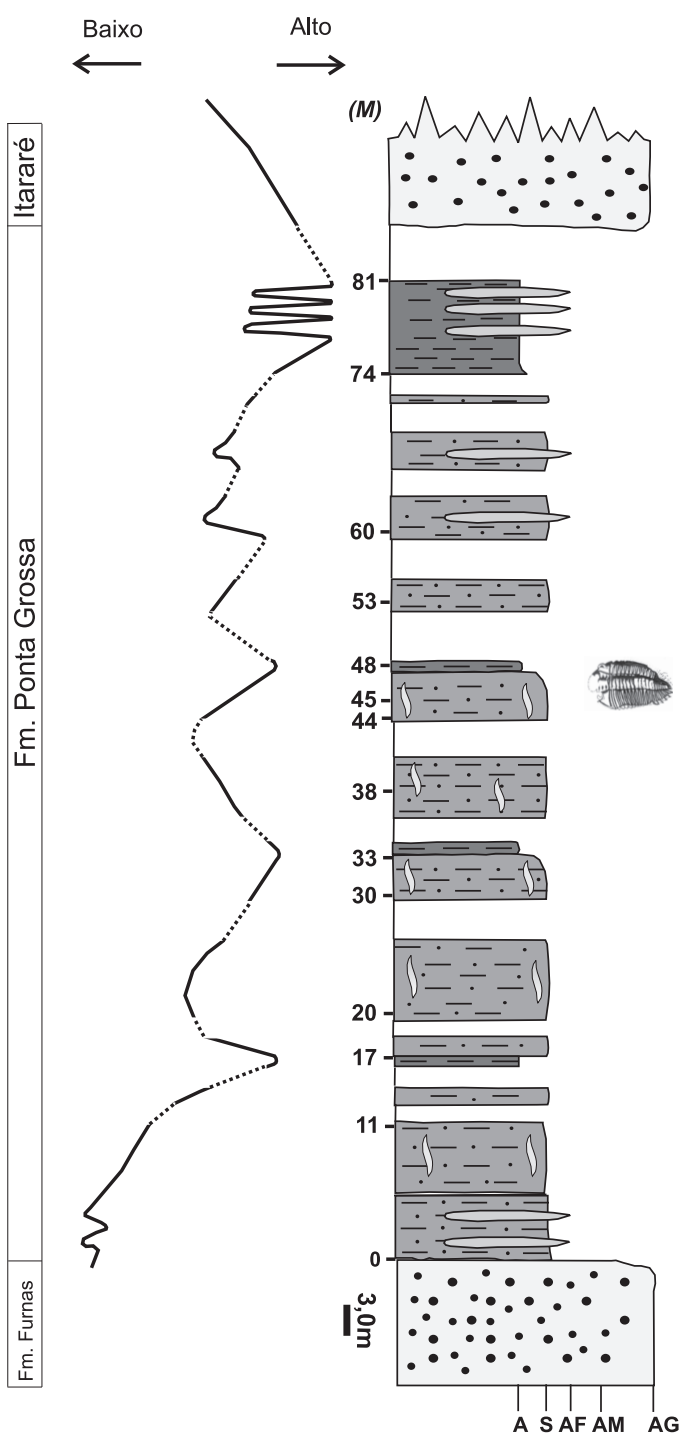

\section{Legenda:}

Argilitos (por vezes folhelhos)

Siltito arenoso a arenito fino

Arenito Médio a Grosso

\section{$\because$}
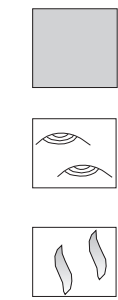

Arenito muito fino

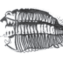

Metacryphaeus rotundatus

Arenito Médio a Grosso

Figura 2. Seções colunares dos afloramentos da Formação Ponta Grossa (= Membro Jaguariaíva). A. Seção colunar composta, incluindo, na base, os litotipos aflorantes ao longo da PR-340, na entrada de Tibagi - PR, bem como da estrada que parte de Tibagi, rumo NW para Telêmaco Borba. B. Seção colunar de Jaguariaíva, ao longo do ramal ferroviário. Seções colunares modificadas de Ghilardi (2004). 


\section{Descrição do material}

Os espécimes aqui descritos pertencem todos à coleção científica da UNESP/Botucatu, cuja preparação foi realizada de acordo com as técnicas usualmente empregadas em estudos paleontológicos de macrofósseis marinhos (Feldmann, Chapman, Hannibal, 1989; Simões e Fittipaldi, 1992; Nobree Carvalho, 2004). Já a revisão sistemática levou em consideração a terminologia morfológica usualmente empregada para os trilobites calmoniídeos (Eldredge e Branisa, 1980; Lieberman, Edgecombe, Eldredge, 1991; Lieberman, 1993). Conforme recomendado por Whittington (1997), os lobos e sulcos glabelares são precedidos dos prefixos $\mathrm{L}$ e $\mathrm{S}$, respectivamente, numerados de 0 (occipital) a 4 (anterior). Os espécimes foram observados sob estereomicroscópio Leica, modelo MZ75. As imagens digitais que ilustram a presente contribuição foram obtidas através de câmera digital acoplada aos estereomicroscópios Zeiss e Leica.

\section{RESULTADOS}

\section{Paleontologia sistemática}

Ordem Phacopida Salter, 1864.

Subordem Phacopina Struve, 1959.

Família Calmoniidae Delo, 1935.

Gênero Metacryphaeus Reed, 1907.

Metacryphaeus rotundatus Kozlowski, 1923 (Figuras 3A e 3B).

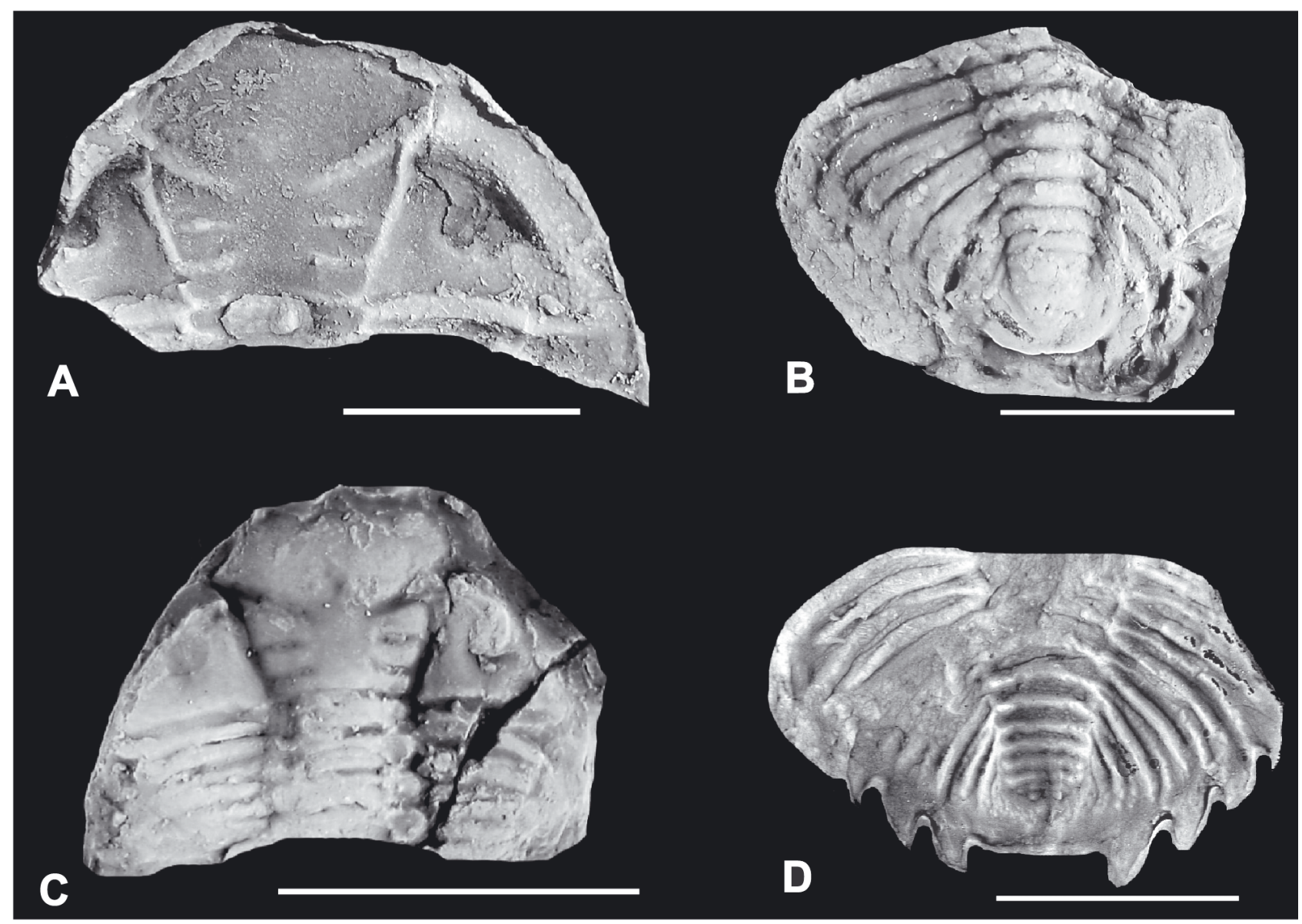

Figura 3. Espécimes de Metacryphaeus rotundatus (A, B) e Metacryphaeus australis (C, D). A. Céfalo de M. rotundatus, mostrando o lobo frontal anteriormente arredondado e espinho genal longo, delgado, comprimido na base, paralelo à borda lateral (DZP-18626). B. Pigídio de M. rotundatus, com as pleuras levemente arqueadas sagitalmente e flexionadas para baixo, lateralmente (DZP-18570a). C. Céfalo de M. australis, com glabela saliente e margem não prolongada anteriormente, (DZP-3706). D. Pigídio de M. australis, subtriangular, mais largo do que longo, margem pigidial subtriangular, com terminação em forma de largas franjas, chatas, agudas, e recurvadas para trás. Terminação do axis pigidial em forma de lapela larga, mais curta do que as das franjas (DZP -3699). Escala gráfica: $1 \mathrm{~cm}$. 
Cryphaeus giganteus Ulrich, 1892, Pl. 1, figura 7.

Cryphaeus australis var. rotundatus Kozlowski, 1923, Pl. 3, figuras 1 e 11 .

Metacryphaeus giganteus (Ulrich, 1892) Branisa, 1965, Pl. 13, figuras 8, 11 e 12.

Descrição: lobo frontal arredondado anteriormente; espinho genal longo, delgado, comprimido na base, paralelo à borda lateral; L3 achatado (tr.); S0 fracamente cortado medialmente; margem cefálica prolongada anteriormente (Figura 3A). Pigídio aproximadamente triangular no contorno, sulco axial reto, convergindo para trás após os cinco anéis anteriores, menos divergentes posteriormente; axis pigidial com 10 anéis e segmento terminal alcançando as últimas pleuras; três anéis anteriores levemente curvos para frente; cinco sulcos anteriores moderadamente profundos medialmente e profundos na confluência com sulcos axiais; divergência entre sulcos axiais diminui a partir do sexto anel em direção posterior; axis estreita-se abruptamente também a partir do sexto anel, acompanhando diminuição da divergência entre sulcos axiais; pleuras com sete pares de sulcos pleurais, sendo cinco pares anteriores largos e moderadamente profundos; dois últimos pares rasos; pleuras levemente arqueadas sagitalmente e flexionadas para baixo lateralmente; pleuras em forma de lapelas, arredondadas (Figura 3B).

Ocorrências e horizontes fossilíferos: Formação Ponta Grossa, Membro Jaguariaíva (Seqüência B). Afloramento localizado no corte da estrada de ferro Jaguariaíva-Arapoti, a $48 \mathrm{~m}$ da base da seção. Afloramentos encontrados no quilômetro 60 da PR-340 (Castro-Tibagi), na entrada de Tibagi e afloramentos a cerca de $3 \mathrm{~km}$ da entrada de Tibagi, na subida da estrada que parte desta cidade em direção NW, rumo a Telêmaco Borba, Estado do Paraná. Os horizontes fossilíferos estão situados, respectivamente, a 8 e 48 m da base da seção.

Material examinado: espécimes IBB/UNESP, DZP 3382; 17095; 17123a, b; 17243; 17257; 17429; 18570; 18581a, b; 18590a, b; 18626.

Discussão: o gênero Metacryphaeus é conhecido para a Bacia do Parnaíba (Carvalho, Edgecombe, Lieberman, 1994; Carvalho, Edgecombe, Lieberman, 1997), Bacia do Paraná (Formação Ponta Grossa e Grupo Chapada; Clarke, 1913; Castro, 1968; Popp, 1985; Carvalho e Quadros, 1987), bem como para as assembléias devonianas da Bolívia (Formações Icla, Belén, Sicasica; Wolfart, 1968) e Ilhas Falklands (Formação Fox Bay; Edgecombe, 1991; Carvalho, 2006) (Tabela 1).

Metacryphaeus rotundatus é aqui descrita pela primeira vez para o Devoniano da Bacia do Paraná. Essa espécie foi reconhecida, primeiramente, por Kozlowski (1923), para exemplares do Devoniano da Bolívia, Formação Icla, pela distinção da forma arredondada dos céfalos, sendo designada como Cryphaeus australis var. rotundatus. Posteriormente, Lieberman (1993) desenvolveu um estudo filogenético dos táxons pertencentes ao "grupo Metacryphaeus", onde foi identificado que $M$. rotundatus seria grupo-irmão de $M$. giganteus, e não de $M$. australis, a partir do compartilhamento do espinho genal longo, delgado e comprimido na base. Portanto, segundo Lieberman (1993) é improvável que $M$. rotundatus seja uma subespécie de M. australis. Adicionalmente, após revisão de Lieberman (1993), foi atribuído o status de espécie para $M$. rotundatus, uma vez que apresenta caracteres que permitem distingui-la de M. australis, principalmente no que diz respeito a feições morfológicas do pigídio, como a forma das lapelas laterais e o tamanho do axis pididial, e do céfalo, como a forma do espinho genal e da margem cefálica.

Dessa forma, os exemplares estudados (DZP- 3382; 17095; 17123a, b; 17243; 17257; 17429; 18570; 18581a, b; 18590a, b; 18626) são aqui atribuídos a M. rotundatus, pois apresentam feições diagnósticas dessa espécie, tais como:

a. axis pigidial prolongando-se em uma terminação que alcança as lapelas laterais (Figura 3B);

b. lapelas laterais arredondadas (Figura 3B);

c. espinho genal longo, delgado, comprimido na base (Figura 3A);

d. margem cefálica prolongada anteriormente (Figura 3A).

Espécimes de M. australis, utilizados para comparação (DZP-3699, 17095; 17243; 17257; 17095; 18570a; 18590a, b) apresentam:

a. axis pigidial terminando antes de alcançar as lapelas laterais (Figura 3D);

b. lapelas laterais agudas (Figura 3D);

c. ausência de espinho genal (Figura 3C);

d. margem cefálica sem prolongamento (Figura 3C).

\section{DISCUSSÃO E COMENTÁRIOS FINAIS}

De acordo com os dados disponíveis de momento, Metacryphaeus rotundatus ocorre em argilitos maciços. A despeito da desarticulação das carapaças, as quais devem representar restos de mudas (vide discussão em Ghilardi, 2004), esses trilobites parecem representar ocorrências autóctones a parautóctones. Indivíduos da espécie Metacryphaeus rotundatus devem ter ocupado, preferencialmente, fundos lamosos, ricos em matéria orgânica, depositados sob condições de águas plataformais, abaixo do nível de base de ondas de tempestades, associados a eventos de inundação marinha. A presença dessa espécie na Formação Ponta Grossa, Sub-bacia Apucarana, indica tratar-se de um elemento com apreciável distribuição paleobiogeográfica, no contexto do Domínio Malvinocáfrico. Isso porque Metacryphaeus rotundatus é uma espécie co- 
Tabela 1. Lista das espécies de calmoniídeos encontrados no Devoniano do Brasil e da Bolívia.

\begin{tabular}{|c|c|c|}
\hline Calmoniidae Brasil & Calmoniidae Bolívia & Autor \\
\hline Bainella pontagrossensis & & Carvalho e Edgecombe, 1991 \\
\hline Calmonia paranaensis & & Popp, 1985 \\
\hline Calmonia signifer var. micrischia & & Clarke, 1913 \\
\hline Calmonia signifier & & Clarke, 1913 \\
\hline Calmonia subseciva & & Clarke, 1913 \\
\hline Calmonia? triacantha & & Carvalho e Edgecombe, 1991 \\
\hline Paracalmonia cuspidata & & Struve, 1958 \\
\hline Paracalmonia mendesi & & Popp, 1996 \\
\hline Paracalmonia paranaensis & & Рopp, 1996 \\
\hline Paracalmonia pessula & & Struve, 1958 \\
\hline Paracalmonia salamunii & & Popp, 1996 \\
\hline Pennaia pauliana & & Clarke, 1913 \\
\hline Tibagya parana & & Struve, 1958 \\
\hline Metacryphaeus granulata & & Popp, 1985 \\
\hline Metacryphaeus sedori & & Popp, 1985 \\
\hline Metacryphaeus australis & Metacryphaeus australis & Struve, 1958 \\
\hline \multirow[t]{34}{*}{ Metacryphaeus rotundatus } & Metacryphaeus rotundatus & Lieberman, 1993; Presente estudo \\
\hline & "Clarkeaspis" gouldi & Lieberman, 1993 \\
\hline & “Palpebrops" goeldi (Kaltzer) & Lieberman, Edgecombe e Eldredge, 1991 \\
\hline & Andinacaste chojnacotensis & Eldredge e Branisa, 1980 \\
\hline & Andinacaste legrandi & Eldredge e Branisa, 1980 \\
\hline & Bainella (Belenops) insólita (Wolfart) & Eldredge e Branisa, 1980 \\
\hline & Clarkeaspis padillaensis & Lieberman, 1993 \\
\hline & Deltacephalaspis (Deltacephalaspis) comis & Eldredge e Branisa, 1980 \\
\hline & Deltacephalaspis (Deltacephalaspis) magister & Eldredge e Branisa, 1980 \\
\hline & Deltacephalaspis (Deltacephalaspis) retrospina & Eldredge e Branisa, 1980 \\
\hline & Deltacephalaspis (Prestalia) túmida & Eldredge e Branisa, 1980 \\
\hline & Eldredgeia venustus & Lieberman, 1993 \\
\hline & Kozlowskiaspis (Kozlowskiaspis) superna & Eldredge e Branisa, 1980 \\
\hline & Kozlowskiaspis (Romanops) australis & Eldredge e Branisa, 1980 \\
\hline & Kozlowskiaspis (Romanops) borealis & Eldredge e Branisa, 1980 \\
\hline & Malvinella buddeae & Lieberman, Edgecombe e Eldredge, 1991 \\
\hline & Malvinella haugi & Lieberman, Edgecombe e Eldredge, 1991 \\
\hline & Malvinocooperella pregiganteus & Lieberman, 1993 \\
\hline & Metacryphaeus branisai & Lieberman, 1993 \\
\hline & Metacryphaeus convexus & Lieberman, 1993 \\
\hline & Metacryphaeus curvigena & Lieberman, 1993 \\
\hline & Metacryphaeus giganteus & Lieberman, 1993 \\
\hline & Metacryphaeus tuberculatus & Lieberman, 1993 \\
\hline & Palpebrops donegalensis & Lieberman, Edgecombe e Eldredge, 1991 \\
\hline & Parabouleia calmonensis & Eldredge, 1972 \\
\hline & Phacopina convexa & Eldredge e Branisa, 1980 \\
\hline & Phacopina padilla & Eldredge e Branisa, 1980 \\
\hline & Plesioconvexa praecursor & Lieberman, 1993 \\
\hline & Plesiomalvinella boulei (Kozlowki) & Lieberman, Edgecombe e Eldredge, 1991 \\
\hline & Plesiomalvinella pujravii (Wolfart) & Lieberman, Edgecombe e Eldredge, 1991 \\
\hline & Schizostylus (Curuyella) granulata & Eldredge e Branisa, 1980 \\
\hline & Tarijactinoides jarcasensis & Suárez Soruco, 1971 \\
\hline & Tarijactinoides tikanensis & Eldredge e Branisa, 1980 \\
\hline & Wolfartaspis cornutus & Lieberman, 1993 \\
\hline
\end{tabular}


mum na fauna devoniana da Bolívia, Formação Icla (Kozlowski, 1923; Lieberman, 1993) (Tabela 1). Desse modo, durante os eventos transgressivos, materializados pelos depósitos de inundação marinha que contêm Metacryphaeus rotundatus, as condições seriam favoráveis à dispersão, pela diminuição das barreiras geográficas. Nesse contexto, Metacryphaeus rotundatus possui distribuição paleobiogeográfica maior do que anteriormente suposto, no contexto do Domínio Malvinocáfrico. Além disso, durante o Devoniano, os calmoniídeos bolivianos viveram em águas situadas em torno dos $45^{\circ}$ a $50^{\circ}$ de latitude sul e os da Bacia do Paraná, próximas aos $70^{\circ}$ de latitude sul (Copper, 1977; Eldredge e Ormiston, 1976). Assim sendo, é sugestivo supor que Metacryphaeus rotundatus deve ter sido uma espécie euritérmica.

Nos depósitos da Formação Icla da Bolívia, Metacryphaeus rotundatus ocorre em rochas do PraguianoEmsiano. Com base em quitinozoários, principalmente na ocorrência de Pterochitina sp., Ramochitina sp., Ramochitina magnífica, Ancyrochitina sp. e Ancyrochitina parisi, Grahn (2002) atribuiu idade praguiana para a base da Formação Icla e eoemsiana a eo-eifeliana, para o topo da Formação Icla. A presença de Metacryphaeus rotundatus em rochas da porção basal da Formação Ponta Grossa, reforça a interpretação de que seus depósitos abrangem o intervalo Praguiano-Emsiano, conforme já apontado por outros grupos de macro e microfósseis.

Conforme demonstrado pela presença de Metacryphaeus rotundatus nos depósitos da Formação Ponta Grossa, a fauna de macroinvertebrados, em especial de trilobites calmoniídeos, é mais diversificada do que até então se supunha. A fauna é, possivelmente, constituída por 17 espécies (Clarke, 1913; Struve, 1958; Popp, 1985; Popp, Coimbra e Hauch, 1996; Carvalho e Edgecombe, 1991), incluindo agora Metacryphaeus rotundatus. Considerando-se os dados presentes nesse estudo e a lista de espécies de trilobites disponível para a Bacia do Paraná (vide Ghilardi e Simões, 2007) (Tabela 1), observa-se que os gêneros Calmonia Clarke (1913), Paracalmonia Reed (1907) e Metacryphaeus Reed (1907) são os mais diversificados, dentre os calmoniídeos da Sub-bacia Apucarana.

Finalmente, embora o estudo paleontológico dos depósitos fossilíferos da Formação Ponta Grossa remonte ao início do século passado, essa unidade continua ainda fornecendo novos dados paleontológicos e estratigráficos que, em seu conjunto, contribuem para o refinamento da composição faunística e afinidades paleobiogeográficas das assembléias fósseis e seu significado paleoecológico e geocronológico. Nesse contexto, seria interessante que pesquisas futuras abrangessem o Devoniano da Bolívia e áreas ainda pouco exploradas (Mato Grosso, Goiás) da Bacia do Paraná.

\section{AGRADECIMENTOS}

A presente pesquisa contou com a participação dos professores: Dra. M.J. Garcia (UNG), Dr. L.E. Anelli (IGc/USP), Dr. E. Bosetti (UEPG) e Dr. R.T. Bolzon (UFPR), aos quais agradecemos a colaboração. Os autores agradecem, especialmente, aos revisores, Prof. Dr. Setembrino Petri (IGc/USP) e Profa. Dra. Rosemarie Rohn Davies (IGCE/UNESP), pelas sugestões, comentários e correções nas versões iniciais desse manuscrito. Finalmente, os autores agradecem ainda à FAPESP (99/11763-6, 05/00791-1) e a PROAP (Programa de Apoio à Pós-graduação da CAPES, IGc/USP), pelo apoio financeiro oferecido.

\section{REFERÊNCIAS BIBLIOGRÁFICAS}

BERGAMASCHI, S. Análise estratigráfica do SiluroDevoniano (Formação Furnas e Ponta Grossa) da subbacia de Apucarana, Bacia do Paraná, Brasil. 1999. 167 f. Tese (Doutorado) - Instituto de Geociências, Universidade de São Paulo, São Paulo, 1999.

BERGAMASCHI, S.; PEREIRA, E. Caracterização de seqüências deposicionais de $3^{\text {a }}$ ordem para o SiluroDevoniano na sub-bacia de Apucarana, bacia do Paraná, Brasil. Ciência Técnica Petróleo. Seção Exploração de Petróleo, v. 20, p.63-72, 2001.

BOSETTI, E. P. Tafonomia de alta resolução das fácies de offshore da sucessão devoniana na região de Ponta Grossa, Paraná, Brasil. 2004. 112 f. Tese (Doutorado) - Universidade Federal do Rio Grande do Sul, Porto Alegre, 2004.

BRANISA, L. Los fosiles guias de Bolivia. Servicio Geologico de Bolivia, Boletín, v. 6, p. 1-282, 1965.

CARVALHO, M. G. P. Devonian trilobites from the Falkland Islands. Palaeontology, v. 49, n. 1, p. 21-34, 2006.

CARVALHO, M. G. P.; EDGECOMBE, G. D. Lower -early middle Devonian calmoniid trilobites from Mato Grosso, Brazil, and related species from Paraná. American Museum Novitates, v. 3022, p. 1-13, 1991.

CARVALHO, M. G. P.; QUADROS, L. P. Trilobitas devonianos do flanco noroeste da Bacia do Paraná. In: CONGRESSO BRASILEIRO DE PALEONTOLOGIA, 10., 1987, Rio de Janeiro. Anais... Rio de Janeiro: SBP, 1987. v. 2, p. 545-565.

CARVALHO, M. G. P.; EDGECOMBE, G. D.; LIEBERMAN, B. S. Devonian Calmoniid Trilobites from Parnaíba Basin, 
Piauí State, Brazil. Anais da Academia Brasileira de Ciências, v. 66, n. 1, p. 119, 1994.

CARVALHO, M. G. P.; EDGECOMBE, G. D.; LIEBERMAN, B. S. Devonian Calmoniid trilobites from the Parnaíba Basin, Piauí State, Brazil. American Museum Novitates, v. 3192, p. 1-11, 1997.

CASTRO, J. S. Trilobitas da Formação Pimenteiras, Devoniano do estado do Piauí. Anais da Academia Brasileira de Ciências, v. 40, n. 4, p. 481-489, 1968.

CLARKE, J. M. Fósseis devonianos do Paraná. Monografia do Serviço Geológico e Mineralógico do Brasil, v. 1, p. 1-353, 1913.

COPPER, P. Paleolatitudes in the Devonian of Brazil and the Frasnian - Famennian mass extinction. Palaeogeography, Palaeoclimatology, Palaeoecology, v. 21, p. 165-207, 1977.

DELO, D. M. A revision of the Phacopid trilobites. Journal of Paleontology, v. 9, p. 402-420, 1935.

DINO, R.; OLIVEIRA, S. F.; ANTONIOLI, L. Análise palinológica de sedimentos da Formação Ponta Grossa aflorantes no flanco NW da Bacia do Paraná, MT, Brasil. In: SIMPÓSIO SOBRE A CRONOESTRATIGRAFIA DABACIA DO PARANÁ, 2., 1995, Porto Alegre. Boletim de Resumos Expandidos... Porto Alegre, 1995. v. 1, p. 1-9.

EDGECOMBE, G. D. Calmoniid trilobites from the Devonian Fox Bay Formation, Falkland Islands. In: LANDING, E. Studies in Paleontology and Stratigraphy in Honor of Donald W. Fisher. Bulletin of the New York State Museum, v. 481, p. 55-68, 1991.

ELDREDGE, N. Morphology and relationships of Bouleia Kozlowski, 1923 (Trilobita, Calmoniidae). Journal of Paleontology, v. 46, p. 140-151, 1972.

ELDREDGE, N.; BRANISA, L. Calmoniid trilobites of the Lower Scaphiocoelia Zone of Bolivia, with remarks on related species. Bulletin of the American Museum of Natural History, v. 165, n. 2, p. 185-289, 1980.

ELDREDGE, N.; ORMISTON, A. R. Biogeography of Silurian and Devonian Trilobites of the Malvinokaffric Realm. In: BOUCOT, A. J.; GRAY, J. Historical Biogeography, Plate Tectonics, and Changing Environment. Corvalis: Oregon State Univ. Press: 1976, p. 147-167.

FELDMANN, R. M.; CHAPMAN, R. E.; HANNIBAL, J. T.
Paleotechiniques. The Paleontological Society. Special Publications, v. 4, 1989. 358 p.

GAUGRIS, K. A.; GRAHN, Y. New chitinozoan species from the Devonian of the Paraná Basin, south Brazil, and their biostratigraphic significance. Ameghiniana, v. 43, n. 2, p. 293-310, 2006.

GHILARDI, R. P. Tafonomia comparada e Paleoecologia dos Macroinvertebrados (ênfase em Trilobites), da Formação Ponta Grossa (Devoniano, Sub-bacia Apucarana), Estado do Paraná, Brasil. 2004. 113 f. Tese (Doutorado)Instituto de Geociências, Universidade de São Paulo, São Paulo, 2004.

GHILARDI, R. P.; SIMÕES, M .G. History and Development of Trilobite Research in Brazil. In: MIKULIC, D. G; LANDING, E.; KLUESSENDORF, J. Fabulous Fossils - 300 years of worldwide research on trilobites. New York State Museum Bulletin. v. 507, p.97-104, 2007.

GRAHN, Y. Upper Silurian and Devonian Chitinozoa from central and southern Bolívia, central Andes. Journal of South American Earth Sciences, v. 15, p. 315-326, 2002.

GRAHN, Y.; PEREIRA, E.; BERGAMASCHI, S. Silurian and Lower Devonian chitinozoan biostratigraphy of the Paraná Basin in Brazil and Paraguay. Palynology, v. 24, p. 147-176, 2000.

KOZLOWSKI, R. Faune Dévonienne de Bolivie. Annales de Paleontologie, v. 12, n. 1/2, p. 1-112, 1923.

LANGE, F. W.; PETRI, S. The Devonian of the Paraná Basin. Boletim Paranaense de Geociências, v. 21-22, p. 5-55, 1967.

LIEBERMAN, B. S. Systematics and biogeography of the “Metacryphaeus Group” Calmoniidae (Trilobita, Devonian), with comments on adaptative radiations and the geological history of the Malvinokaffric Realm. Journal of Paleontology, v. 67, n. 4, p. 549-570, 1993.

LIEBERMAN, B. S.; EDGECOMBE, G. D.; ELDREDGE, N. Systematics and biogeography of the "Malvinella Group", Calmoniidae (Trilobita, Devonian). Journal of Paleontology, v. 65, n. 5, p. 824-843, 1991.

NOBRE, P. H.; CARVALHO, I. S. Fósseis: coleta e métodos de estudo. In: CARVALHO, I. S. Paleontologia. Rio de Janeiro: Interciência, 2004, p. 27-42.

PETRI, S. Contribuição ao estudo do Devoniano paranaense. 
Boletim do Departamento Nacional de Produção Mineral, v. 129, p. 1-125, 1948.

PETRI, S. Problems in the utilization of the Brazilian Code of Stratigraphic Nomenclatures as related to modern researches. Revista Brasileira de Geociências, v. 36, n. 1, p. 203-208, 2006.

POPP, M. T. B. Revisão dos trilobitas Calmoniideos e comunidades faunísticas da formação Ponta Grossa, Devoniano, no estado do Paraná. 1985. 112 f. Tese (Doutorado) - Universidade Federal do Rio Grande do Sul, Porto Alegre, 1985.

POPP, M. T. B.; COIMBRA, J. C.; HAUCH, A. Revisão do gênero Paracalmonia Struve, 1958 (Proboloides Clarke, 1913), Trilobita - um ensaio de sistemática filogenética. Gaia, v. 12, p. 19-32, 1996.

REED, F. R. C. The Fauna of Bokkeveld Beds. Geological Magazine, v. 4, p. 165-232, 1907.

RODRIGUES, S. C.; LEME, J. M.; SIMÕES, M. G. Tafonomia Comparada dos Conulatae (Cnidaria), Formação Ponta Grossa (Devoniano), Bacia do Paraná, Estado do Paraná. Revista Brasileira de Geociências, v. 4, n. 33, p.1-10, 2003.

SALTER, J. W. British fossils. Memoirs of the Geological Survey of Great Britain. Decade 11, 1864.

SIMÕES, M. G.; FITTIPALDI, F. C. Fósseis da região de Rio Claro, SP. Arquivo do Município de Rio Claro, $1^{\text {a }}$ Edição, p. 1-77, 1992.

SIMÕES, M. G.; GHILARDI, R. P.; SALES, A. M. F.; RODRIGUES, S. C.; LEME, J. M.; MELLO, L. H. C. Taphonomy and Gênesis of the "Conularia Beds" from Ponta Grossa Formation (Devonian) within a Sequence Stratigraphy Framework. In: PALEO/2000, SP, 2000, Botucatu, Boletim de Resumos... Botucatu, 2000a, p. 20.

SIMÕES, M. G; MELLO, L. H. C.; RODRIGUES, S. C.; LEME, J. M.; MARQUES, A. C. Conulariid taphonomy as a tool in paleoenvironmental analysis. Revista Brasileira de Geociências. Brasília, v. 4, n. 30, p. 757-762, 2000 b.

STRUVE, W. Uber einige Fälle von Homonymie bei Gattungen der Phacopacea. Senckenbergiana Lethaea, v. 39, p. 301-302, 1958.

STRUVE, W. Trilobita. In: MOORE, R. C. Treatise on Invertebrate Paleontology Part O. Arthropoda. Boulder,
CO; Lawrence, KS: Geological Society of America: University of Kansas, 1959, p. O483-O489.

SUÁREZ-SORUCO, R. Tarijactinoides jarcasensis n. gen. n. sp. del Devoniano Inferior. Servicio Geologico Bolivia Bulletin, v. 15, p. 53-56, 1971.

ULRICH, A. Palaeozoische Versteinerungen aus Bolivien. In: STEINMANN, G. Beiträge zur Geologie und Paläontologie von Südamerika. Stuttgart: Schweizerbart, 1892, p. 1-116.

WHITTINGTON, H. B. Morphology of the Exoeskeleton. In: KAESLER, R. L. Treatise on Invertebrate Paleontology Part O. Arthropoda1, Trilobita, Revised. Boulder: Geological Society of America, 1997, p. O1-O85.

WOLFART, R. Die Trilobite naus dem Devon Boliviens und ihre Bedeutung für stratigraphie und tiergeographie. In: WOLFART, R.; VOGES, A. Beitrage zur Kenntnis des Devons von Bolivien. Beihefte zum Geologischen Jahrbuch, v. 74, p. 5-201, 1968 . 